\title{
TRAPPED MODES OF INTERNAL WAVES IN A CHANNEL SPANNED BY A SUBMERGED CYLINDER
}

\author{
by \\ Nikolay Kuznetsov \\ Laboratory on Mathematical Modelling in Mechanics \\ Institute of Problems of Mechanical Engineering, Russian Academy of Sciences \\ Bol'shoy pr., 61, V.O., 199178, St. Petersburg, Russia.
}

\begin{abstract}
A horizontal channel of infinite length and depth and of constant width contains inviscid, incompressible, two-layer fluid under gravity. The upper layer has constant finite depth and is occupied by a fluid of constant density $\rho^{*}$. The lower layer has infinite depth and is occupied by a fluid of constant density $\rho>\rho^{*}$. The parameter $\epsilon=\left(\rho / \rho^{*}\right)-1$ is assumed to be small. The lower fluid is bounded internally by an immersed horizontal cylinder which extends right across the channel and has its generators normal to the sidewalls. The free, time-harmonic oscillations of fluid, which have finite kinetic and potential energy (such oscillations are called trapped modes), are investigated. Trapped modes in homogeneous fluid above submerged cylinders and other obstacles are well known (see Ursell $(1951,1987)$, Jones (1953), McIver \& Evans (1985), Aranha (1988), Bonnet \& Joly (1990), Callan (1990) and references cited in these papers). In the present paper it is shown that there are two sets of frequencies of trapped modes for the two-layer fluid. The frequencies of the first finite set are close to the frequencies of trapped modes in the homogeneous fluid (when $\rho^{*}=\rho$ ). They correspond to the trapped modes of waves on the free surface of upper fluid. The frequencies of the second finite set are proportional to $\epsilon$, and hence, are small. The last frequencies correspond to the trapped modes of internal waves on the interface between two fluids. To obtain these results the perturbation method for a square operator pencil was applied. The square operator pencil with bounded, symmetric, linear, integral operators in the space $L_{2}(-\infty,+\infty)$ arises as a result of two reductions of the original problem. The first reduction allows to consider only the potential in the lower fluid. The second reduction is the same that was used by Ursell (1987).
\end{abstract}

\section{Introduction}

Trapped modes of waves on the free surface of a homogeneous fluid are well known. By trapped modes we mean the free, time-harmonic oscillations of the fluid decaying to zero at infinity. In the case of modes trapped in a channel waves have finite kinetic and potential energy. The first explicit solution for trapped modes of, so called, edge waves was discovered by Stokes (1846). He showed that a wave may progress over a sloping beach along a straight 
coastline with the motion decaying exponentially in the offshore direction. The existence of trapped modes in a channel above a submerged horizontal cylinder spanning sidewalls was first established by Ursell (1951). He proved that there is a finite set of frequencies of trapped waves below a certain cutoff frequency, if a circular cylinder of a sufficiently small radius is immersed in deep water. Soon after that, Jones (1953) generalized Ursell's result to cylinders of arbitrary but symmetric cross-section, and finite water depth. In 1987 Ursell gave the new proof of these theorems. Etsensive numerical results was obtained by McIver \& Evans (1985) for trapped modes above circular submerged cylinder. They found that only single mode exists for a depth of submergence of the cylinder greater than about 1.07 of its radius. As the depth of submergence is decreased, further trapped modes appear. Martin (1989) also investigated trapped modes numerically.

Trapped modes also exist if there is a crest on the channel bottom (see Jones (1953), Bonnet \& Joly (1990) and references in the last paper). However, in the case of a hole in the channel bottom trapped modes do not exist as was noticed by Vainberg \& Maz'ya (1973). McIver (1991) proved that there are no trapped waves if the cylinder is semiimmersed and satisfies John's condition. This result can be improved using the version of Maz'ya's identity for surface-piercing bodies suggested by Kuznetsov (1991). In particular, trapped modes are absent not only for cylinders, but for arbitrary, non-necessarily spanning sidewalls, semiimmersed bodies, including those extending throughout the water depth, which satisfy John's condition and have water-line without tangents parallel to the sidewalls. On the contrary, Callan, Linton \& Evans (1991) proved that for circular cylinder placed symmetrically between the sidewalls and extending throughout the water depth the trapped modes exist (the water-line of such a cylinder has two tangents parallel to the sidewalls).

One can see that the question on trapped modes in homogeneous fluid is investigated rather well. At the same time the author has no information about any paper treating the trapped modes problem for a two-layer fluid. The importance of such investigations can be recognized from the following remark by Friis, Grue \& Palm (1991):

"Long underwater tube bridges [...] are proposed to be constructed across Norwegian fjords and straits", since it is well known that these fjords often are occupied by two-layer fluid. The upper layer contains fresh water and the lower layer contains salt water which has density a slightly greater, than the fresh water.

Now, the contents of the paper will be briefly observed. In Section 1 the statement of the problem is given. Section 2 is devoted to the reduction of the original problem to the problem which has only one unknown potential, that describes motion in the lower fluid. This is performed with the help of the Fourier transform. The next Section 3 contains the second step of the reduction. Using the method developed by Ursell (1987) and based on the special Green function we get a square operator pencil. The operators involved are bounded, symmetric, integral operators in the space $L_{2}(-\infty,+\infty)$. Their properties are investigated in Appendix. There is a small parameter in the pencil, since it is assumed that the difference between the densities of lower and upper fluid is small. Then it is reasonable to apply perturbation method. It is all the more convenient because the unperturbed square operator pencil is degenerate and one of its eigenvalues is equal to zero. The perturbation procedure is developed in Section 4. As a result we find under 
some restrictions, that the square operator pencil has two finite sets of positive eigenvalues.

There is a simple illustration which makes the results of Section 4 very clear. Let us consider the square equation

$$
\left(a_{0}+\epsilon a\right) x^{2}-b(1+\epsilon) x+\epsilon=0
$$

where $a_{0}, a, b>0$ and $\epsilon$ is a small positive parameter. The limit equation (as $\epsilon \rightarrow 0$ )

$$
a_{0} x^{2}-b x=x\left(a_{0} x-b\right)=0
$$

has two roots

$$
x_{0}^{(0)}=0, \quad x_{+}^{(0)}=b / a_{0} .
$$

The expansions of the roots of the perturbed equation are

$$
\begin{aligned}
& x_{0}=\frac{1}{b} \epsilon+O\left(\epsilon^{2}\right) \\
& x_{+}=\frac{b}{a_{0}}+\frac{b^{2}\left(a_{0}-a\right)-a_{0}}{a_{0}^{2} b} \epsilon+O\left(\epsilon^{2}\right) .
\end{aligned}
$$

Then these roots are positive, if $\epsilon$ is sufficiently small.

The results of Section 4 are essentially similar to this example. The perturbed square pencil has two finite sets of positive eigenvalues $\left\{\nu^{(+)}\right\}$and $\left\{\nu^{(0)}\right\}$. The first set is generated by positive eigenvalues of the unperturbed pencil. The second set is generated by the zero eigenvalue of the unperturbed pencil. The Section 5 contains a discussion of the hydrodynamical meaning of these eigenvalues. The set $\left\{\nu^{(+)}\right\}$gives the frequencies of trapped modes of waves on the free surface of upper layer. These frequencies are close to the frequencies of trapped modes of waves on the surface of homogeneous fluid without interface. The frequencies of the second set $\left\{\nu^{(0)}\right\}$ are proportional to the small parameter and correspond to the trapped modes of internal waves on the interface between two layers. There is a rough but informative estimate of a quotient of frequencies of trapped modes of internal and surface waves in Section 5.

A rigorous mathematical justification of the perturbation procedure from Section 4 can be obtained by means of some results in general perturbation theory for linear operators in Hilbert space (see, e.g., Friedrichs (1965), Kato (1966)). This theorems will be published in another paper.

\section{Statement of the problem}

We consider a channel of infinite (for the sake of simplicity) depth with vertical sidewalls. It is occupied by two-layer fluid. Fresh wather with density $\rho^{*}$ occupies upper layer which depth (without loss of generality) can be assumed to be equal to one.

We choose the $x y z$-coordinates with the $y$-axis directed upwards and with the $x z$-plane coinciding with the undisturbed interface between two layers (see Figure). The sidewalls lie in the planes $\{z= \pm b\}$. 


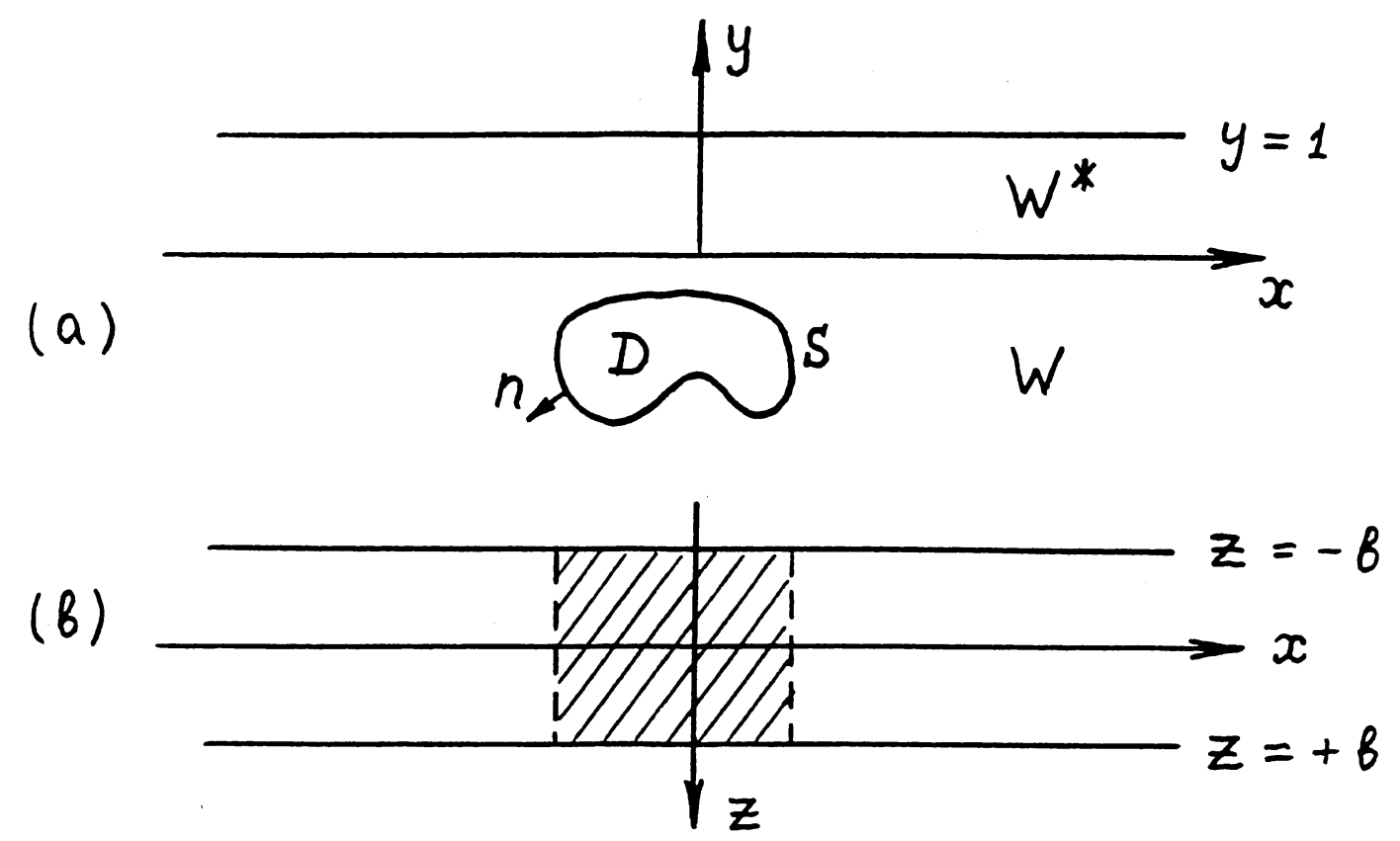

Figure. (a) Cross-section of the channel, (b) view from above.

Salt water with density $\rho>\rho^{*}$ occupies the lower part of the channel and contains a cylinder spanning the sidewalls. It has constant cross-section $D$, and its generators are parallel to the $z$-axis (see Figure).

We shall use the linear theory of surface waves and we assume that $\epsilon=\rho / \rho^{*}-1$ is a samll parameter. We denote by $\phi(x, y, z, t)\left(\phi^{*}(x, y, z, t)\right)$ time-depending velocity potential for lower solt (upper fresh) water. These potentials must satisfy the following relations:

$$
\left.\begin{array}{c}
\nabla^{2} \phi^{*}=0 \text { in } W^{*}, \quad \nabla^{2} \phi=0 \text { in } W \\
\phi_{z}^{*}=0 \text { when } z= \pm b, \quad \phi_{z}=0 \text { when } z= \pm b \\
\phi_{t t}^{*}+g \phi_{y}^{*}=0 \text { when } y=1, \quad \partial \phi / \partial n=0 \text { on } S
\end{array}\right\}
$$

Here $g$ is the acceleration due to gravity and $n$ is the unit normal to the cylinder surface $S$ directed into $W$. The written relations are usual in the linear surface waves theory. We mention only that the last two conditions describe the continuty of the normal component of velocity field and of the pressure across the interface.

Solutions of (1), (2) corresponding to waves of radian frequency $\omega$ and of wavenumber $k$ along the $z$-axis have the form

$$
\left.\begin{array}{l}
\phi(x, y, z, t)=\exp (-i \omega t) u(x, y) \cos k z \\
\phi^{*}(x, y, z, t)=\exp (-i \omega t) u^{*}(x, y) \cos k z
\end{array}\right\}
$$

To satisfy the boundary conditions on sidewalls we have to put

$$
k=\pi n / b, \quad n=1,2,3, \ldots
$$


We can consider the factor $\sin k z$ instead of $\cos k z$ in (3). In what follows we suppose $k$ to be prescribed parameter, but its value is an arbitrary positive number.

Substituting (3) into (1) and (2), we obtain

$$
\left.\begin{array}{c}
\left.u_{x x}^{*}+u_{y y}^{*}=k^{2} u^{*} \text { in } W^{*}, \quad \begin{array}{l}
u_{x x}+u_{y y}=k^{2} u \\
\text { when } y=1, \quad \partial u / \partial n=0 \text { on }
\end{array}\right\} \\
\left.\begin{array}{r}
u_{y}^{*}-\nu u=0 \\
u_{y}^{*}=u_{y} \text { when } y=0
\end{array}\right\} \\
\rho^{*}\left(u_{y}^{*}-\nu u\right)=\rho\left(u_{y}-\nu u\right) \text { when } y=0
\end{array}\right\}
$$

where $\nu=\omega^{2} / g$.

For trapped-mode solutions the motion must decay at large distances

$$
\begin{aligned}
& u^{*},\left|\nabla u^{*}\right| \rightarrow 0 \text { as }|x| \rightarrow \infty \\
& u,|\nabla u| \rightarrow 0 \text { as }|z| \rightarrow \infty \quad(z=x+i y)
\end{aligned}
$$

More precisely, kinetic and potential energy of surface and internal waves should be finite.

$$
\begin{gathered}
\int_{-\infty}^{+\infty}\left\{\left[u^{*}(x, 0)\right]^{2}+\left[u^{*}(x, 1)\right]^{2}\right\} d x+\int_{W^{*}}\left|\nabla u^{*}\right|^{2} d x d y<\infty \\
\int_{-\infty}^{+\infty} u^{2}(x, 0) d x+\int_{W}|\nabla u|^{2} d x d y<\infty
\end{gathered}
$$

Thus, we have the homogeneous boundary value problem with the sepctral parameter $\nu$, which enters into the conditions on the free surface of upper fluid and on the interface. The last condition contains both unknown functions $u$ and $u^{*}$. Our aim is to reduce the problem with two unknown functions to the problem, which can be treated by usual tools of the spectral theory of linear operators (see Kato, 1966). The above mentioned condition on the interface will play the essential role in the reduction, which first setp will be made in the next Section.

\section{Reduction to the problem in the lower layer}

Let us reduce the problem (4)-(6) with unknown pair $\left(u^{*}, u\right)$ to the problem with one unknown function $u$. We have to determine $u^{*}$ assuming that $u_{y}(x, 0)$ is given in the first equation (5).

Using the Fourier transform

$$
\tilde{u}^{*}(\xi, y)=\int_{-\infty}^{+\infty} u^{*}(x, y) e^{-i x \xi} d x
$$

we get

$$
\tilde{u}_{y y}^{*}=\left(k^{2}+\xi^{2}\right) \tilde{u}^{*}, \quad 0<y<1
$$




$$
\begin{gathered}
\tilde{u}_{y}^{*}-\nu \tilde{u}^{*}=0, \quad y=1 \\
\tilde{u}_{y}^{*}=\tilde{u}_{y} \quad, y=0
\end{gathered}
$$

The general solution of the equation (7) is

$$
\tilde{u}^{*}(\xi, y)=C_{1}(\xi) \cosh \lambda y+C_{2}(\xi) \sinh \lambda y, \quad \lambda=\left(k^{2}+\xi^{2}\right)^{1 / 2}
$$

Conditions (8) and (9) yield the system

$$
\left\{\begin{array}{l}
(\lambda \sinh \lambda-\nu \cosh \lambda) C_{1}-(\nu \sinh \lambda-\lambda \cosh \lambda) C_{2}=0 \\
\lambda C_{2}=\tilde{u}_{y}
\end{array}\right.
$$

Hence,

$$
C_{1}(\xi)=\frac{\tilde{u}_{y}}{\lambda} \frac{\nu \sinh \lambda-\lambda \cosh \lambda}{\lambda \sinh \lambda-\nu \cosh \lambda}, \quad C_{2}(\xi)=\frac{\tilde{u}_{y}}{\lambda}
$$

and

$$
\tilde{u}^{*}(\xi, y)=\frac{\tilde{u}_{y}(\xi, 0)}{\lambda} \frac{\nu \sinh \lambda(1-y)-\lambda \cosh \lambda(1-y)}{\lambda \sinh \lambda-\nu \cosh \lambda}
$$

Applying the Fourier transform to the second equation (5) and taking into account the first equation (5), we obtain

$$
\epsilon \tilde{u}_{y}=\nu\left[(1+\epsilon) \tilde{u}-\tilde{u}^{*}\right], \quad y=0
$$

We substitute (10) into the last equality and arrive at

$$
\tilde{u}_{y}=\frac{(1+\epsilon) \nu \lambda(\lambda \tanh \lambda-\nu)}{\left(\nu^{2}+\epsilon \lambda^{2}\right) \tanh \lambda-(1+\epsilon) \nu \lambda} \tilde{u}, \quad y=0
$$

Thus, the problem for $u$ includes

$$
\begin{gathered}
u_{x x}+u_{y y}=k^{2} u \text { in } W \\
\partial u / \partial n=0 \text { on } S \\
u_{y}=\frac{(1+\epsilon) \nu}{2 \pi} \int_{-\infty}^{+\infty} e^{i x \xi} \frac{\lambda(\lambda \tanh \lambda-\nu)}{\left(\nu^{2}+\epsilon \lambda^{2}\right) \tanh \lambda-(1+\epsilon) \nu \lambda} \tilde{u} d \xi \quad \text { when } y=0
\end{gathered}
$$

The trapped-mode solution must also satisfy (6). In (13) we have a pseudo-differential operator depending on the spectral parameter $\nu$, and parameters $k, \epsilon$ which we consider to be prescribed. Moreover, $\epsilon$ is a small paprmeter.

It should be pointed out that the problem (11)-(13), (6) is equivalent to the original problem (4)-(6).

\section{Reduction to the spectral problem for a square operator pencil}

Following to the method developed by Ursell (1987) we use the Green function $g(x, y ; \sigma, 0)$ which satisfies the modified Helmholtz equation (11) with the boundary conditions (12) and 


$$
\begin{gathered}
g_{y}=0 \text { when } y=0 \text { and } x \neq \sigma \\
g \rightarrow 0 \text { as } x^{2}+y^{2} \rightarrow \infty
\end{gathered}
$$

Futhermore, the function $g$ has a source singularity at $(\sigma, 0)$, i.e.

$$
\sup \left\{g(x, y ; \sigma, 0)-K_{0}\left(k\left[(x-\sigma)^{2}+y^{2}\right]^{1 / 2}\right)\right\}<\infty
$$

Here $K_{0}$ is the McDonald function which has the representation

$$
K_{0}(z)=\int_{0}^{\infty} \exp (-z \cosh \mu) d \mu
$$

and the following behaviour

$$
\begin{aligned}
& K_{0}(z) \sim e^{-z}\left(\frac{\pi}{2 z}\right)^{1 / 2} \text { as } z \rightarrow \infty \\
& K_{0}(z) \sim-\log z \text { as } z \rightarrow 0
\end{aligned}
$$

Ursell (1987) proved that $g$ exists, and it can be obtained in the form

$$
\begin{aligned}
& g(x, y ; \sigma, 0)=K_{0}\left(k\left[(x-\sigma)^{2}+y\right]^{1 / 2}\right)+\int_{S} m(s, \sigma)\left\{K_{0}\left(k\left[(x-X(s))^{2}+(y-Y(s))^{2}\right]^{1 / 2}\right)\right. \\
& \left.\quad+K_{0}\left(k\left[(x-X(s))^{2}+(y+Y(s))^{2}\right]^{1 / 2}\right)\right\} d s
\end{aligned}
$$

Here $s$ is the arc length along $S$, while the points of $S$ have coordinates $X(s), Y(s)$. The function $m(s, \sigma)$ is the unique solution of the Fredholm equation of the second kind

$$
\begin{aligned}
& -\pi m\left(s^{\prime}, \sigma\right)+\int_{S} m(s, \sigma) \frac{\partial}{\partial n\left(s^{\prime}\right)}\left\{K_{0}\left(k\left[\left(X\left(s^{\prime}\right)-X(s)\right)^{2}+\left(Y\left(s^{\prime}\right)-Y(s)\right)^{2}\right]^{1 / 2}\right)\right. \\
& \left.+K_{0}\left(k\left[\left(X\left(s^{\prime}\right)-X(s)\right)^{2}+\left(Y\left(s^{\prime}\right)+Y(s)\right)^{2}\right]^{1 / 2}\right)\right\} d s \\
& =-\frac{\partial}{\partial n\left(s^{\prime}\right)} K_{0}\left(k\left[\left(X\left(s^{\prime}\right)-\sigma\right)^{2}+Y^{2}\left(s^{\prime}\right)\right]^{1 / 2}\right)
\end{aligned}
$$

where $\sigma \in(-\infty,+\infty)$ is a parameter.

To reduce the problem (6), (11)-(13) to the spectral problem for a square operator pencil we seek $u$ in the form

$$
u(x, y)=(V \mu)(x, y)=\frac{1}{\pi} \int_{-\infty}^{+\infty} \mu(\sigma) g(x, y ; \sigma, 0) d \sigma
$$

where $\mu \in L_{2}(-\infty,+\infty)$. The single layer Green potential (17) evidently satisfies the equation (11), boundary condition (12) and the condition at infinity (6). Moreover we have from (14) and (16) 


$$
\frac{\partial V \mu}{\partial y}=\mu \text { when } \quad y=0
$$

Substituting the potential (17) into the condition (13), we get in view of (18)

$$
\mu=\frac{(1+\epsilon) \nu}{2 \pi} \int_{-\infty}^{+\infty} e^{i \Sigma \xi} \frac{\lambda(\lambda \tanh \lambda-\nu)}{\left(\nu^{2}+\epsilon \lambda^{2}\right) \tanh \lambda-(1+\epsilon) \nu \lambda}(\widetilde{V \mu}) d \xi \quad \text { when } y=0
$$

Here

$$
\begin{aligned}
& (V \mu)(x, 0)=\pi^{-1} \int_{-\infty}^{+\infty} \mu(\sigma)\left\{K_{0}(k|x-\sigma|)+M(x, \sigma)\right\} d \sigma \\
& M(x, \sigma)=2 \int_{S} m(s, \sigma) K_{0}\left(k\left[(x-X(s))^{2}+Y^{2}(s)\right]^{1 / 2}\right) d s
\end{aligned}
$$

Application of the Fourier transform to (19) gives

$$
\tilde{\mu}(\xi)=\frac{(1+\epsilon) \nu \lambda(\lambda \tanh \lambda-\nu)}{\left(\nu^{2}+\epsilon \lambda^{2}\right) \tanh \lambda-(1+\epsilon) \nu \lambda}(\widetilde{V \mu})(\xi, 0)
$$

This relation can be written in the form of the square operator pencil with respect to the spectral parameter $\nu$. We have

$$
\nu^{2}\left[\lambda^{-2} \tilde{\mu}+(1+\epsilon)\left(\lambda^{-1} \operatorname{ctnh} \lambda\right)(\widetilde{V \mu})\right]-\nu(1+\epsilon)\left[\left(\lambda^{-1} \operatorname{ctnh} \lambda\right) \tilde{\mu}+(\widetilde{V \mu})\right]+\epsilon \tilde{\mu}=0
$$

If we apply the inverse Fourier transform, then we arrive at the square pencil

$$
\begin{aligned}
& \nu^{2}\left[(2 k)^{-1} \int_{-\infty}^{+\infty} \exp (-k|x-\sigma|) \mu(\sigma) d \sigma+(1+\epsilon) \int_{-\infty}^{+\infty} \mathrm{E}(k|x-\sigma|)(V \mu)(\sigma, 0) d \sigma\right] \\
& -\nu(1+\epsilon)\left[\int_{-\infty}^{+\infty} \mathrm{E}(k|x-\sigma|) \mu(\sigma) d \sigma+(V \mu)(x, 0)\right]+\epsilon \mu(x)=0
\end{aligned}
$$

Here we used the relation between the convolution operator and the Fourier transform (see, e.g., Vladimirov, 1971) and well-known formula formula (see, e.g., Gradshtein \& Ryzhik, 1965)

$$
\int_{0}^{\infty} \frac{\cos x \xi}{k^{2}+\xi^{2}} d \xi=\frac{\pi}{2 k} e^{-k|x|}
$$

Also, we introduced the kernel

$$
\mathrm{E}\left(k[x \mid)=\frac{1}{\pi} \int_{0}^{\infty} \frac{\cos x \xi}{\left(k^{2}+\xi^{2}\right)^{1 / 2}} \operatorname{ctnh}\left(k^{2}+\xi^{2}\right)^{1 / 2} d \xi\right.
$$

Below, we shall employ the following notations for the operators involved in (21)

$$
A=L V, \quad A_{0}=A+C, \quad B=L+V
$$

where 


$$
\begin{gathered}
L \mu=(L \mu)(x)=\int_{-\infty}^{+\infty} \mathrm{E}(k|x-\sigma|) \mu(\sigma) d \sigma \\
V \mu=(V \mu)(x, 0) \quad(\operatorname{see}(20)) \\
C \mu=(C \mu)(x)=(2 k)^{-1} \int_{-\infty}^{+\infty} \exp (-k|x-\sigma|) \mu(\sigma) d \sigma
\end{gathered}
$$

All these operators we consider in the space $L_{2}(-\infty,+\infty)$. Their properties are described in Appendix.

The spectral problem for the square operator pencil (21) is equivalent to the boundary value problem (11)-(13), (6) which contains the spectral parameter in the boundary condition (13) on the interface.

\section{Perturbation method for the eigenvalue problem}

In the previous section we obtained the spectral problem for the square operator pencil in $L_{2}(-\infty,+\infty)$

$$
\nu^{2}\left(A_{0}+\epsilon A\right) \mu-\nu(1+\epsilon) B \mu+\epsilon \mu=0
$$

Since there is the small parameter $\epsilon$ in the left hand side, then this operator pencil will be called the perturbed pencil. The unperturbed one is

$$
\nu^{2} A_{0}-\nu B=\nu\left(\nu A_{0}-B\right)
$$

This pencil is degenerate. Obviously, it has an eigenvalue $\nu=0$. Other eigenvalues should be determined from

$$
\nu A_{0} \mu-B \mu=0
$$

The spectral problem (24) is another form of the usual problem about trapped modes in the channel with homogeneous fluid of density $\rho$. The fluid has infinite depth, the mean level of the free surface coincides with $y=1$, and the cylinder is submerged to the depth which is strictly greater than one.

To recognize such a character of (24) one has to return to the boundary value problem (4)-(6). If $\epsilon=0$, then from (5) we get.

$$
u_{y}^{*}=u_{y} \quad \text { and } \quad u^{*}=u \text { when } y=0
$$

Hence $u^{*}$ is the unique continuation of $u$ to the strip $W^{*}$. After such a continuation the function $u$ is a solution of the folowing boundary value problem

$$
\begin{gathered}
u_{x x}+u_{y y}=k^{2} u \quad \text { in } \quad W^{*} \cup(\bar{W} \backslash \bar{D}) \\
u_{y}-\nu u=0 \quad \text { when } \quad y=1
\end{gathered}
$$




$$
\begin{array}{r}
\partial u / \partial n=0 \text { on } S \\
\int_{-\infty}^{+\infty} u^{2}(x, 1) d x+\int_{W \cdot \cup(\bar{W} \backslash \bar{D})}|\nabla u|^{2} d x d y<\infty
\end{array}
$$

Thus, the spectral problem (24) is equivalent to (25)-(28) with the spectral parameter in (26). This problem was investigated in details by Ursell (1987). He reduced (25)-(28) to the spectral problem for a bounded, symmetric, integral operator on the free surface and proved that there are always a finite number of positive point eigenvalues below a certain cutoff frequency. Hence, the problem (24) has the same spectral properties. However, (24) differs in form from the spectral problem that arised in Ursell's paper (1987), because our integral operators are defined on the immersed horizontal line instead of the free surface.

Since, there is the small parameter $\epsilon$ in (22), then it is convenient to apply usual perturbation technique. Let us represent the eigenvalue and the eigenfunction in the form (see, e.g., Friedrichs, 1965)

$$
\left.\begin{array}{l}
\nu=\nu_{0}+\epsilon \nu_{1}+\epsilon^{2} \nu_{2}+\ldots \\
\mu=\mu_{0}+\epsilon \mu_{1}+\epsilon^{2} \mu_{2}+\ldots
\end{array}\right\}
$$

Substituting these expansions into (22) and equating the expressions multiplied by $\epsilon^{0}, \epsilon^{1}$, $\epsilon^{2}, \ldots$ respectively, we obtain the system

$$
\begin{gathered}
\nu_{0}\left(\nu_{0} A_{0} \mu_{0}-B \mu_{0}\right)=0 \\
\nu_{0}\left(\nu_{0} A_{0} \mu_{1}-B \mu_{1}\right)=\left(\nu_{1}+\nu_{0}\right) B \mu_{0}-\mu_{0}-\nu_{0}\left(2 \nu_{1} A_{0} \mu_{0}+\nu_{0} A \mu_{0}\right) \\
\nu_{0}\left(\nu_{0} A_{0} \mu_{2}-B \mu_{2}\right)=\left(\nu_{2}+\nu_{1}\right) B \mu_{0}+\left(\nu_{1}+\nu_{0}\right) B \mu_{1}-\mu_{1}-\nu_{1}^{2} A_{0} \mu_{0} \\
-2 \nu_{0}\left(\nu_{2} A_{0} \mu_{0}+\nu_{1} A \mu_{0}\right)-\nu_{0}\left(2 \nu_{1} A_{0} \mu_{1}+\nu_{0} A \mu_{1}\right)
\end{gathered}
$$

The equation (30) contains the unperturbed operator pencil (23), which has the zero eigenvalue and the finite set of positive eigenvalues satisfying (24) as was pointed out above.

Let us first construct the expansions (29), starting from the non-degenerate positive eigenvalue $\nu_{0}^{(+)}$, and from the corresponding eigenfunction of $(24) \mu_{0}^{(+)}$. The eigenvalue is nondegenerate if the equation

$$
\nu_{0}^{(+)} A_{0} \mu-B \mu=f
$$

has a solution for every right hand side $f$, which is orthogonal to $\mu_{0}^{(+)}$

$$
\left(f, \mu_{0}^{(+)}\right)=\int_{-\infty}^{+\infty} f(x) \mu_{0}^{(+)}(x) d x=0
$$


We assume that $\mu_{0}^{(+)}$is normalized

$$
\left\|\mu_{0}^{(+)}\right\|^{2}=\int_{-\infty}^{+\infty}\left[\mu_{0}^{(+)}(x)\right]^{2} d x=1
$$

It is clear, that the perturbed eigenfunction $\mu^{(+)}$cannot be found from (30)-(32),.. uniquely, since we can multiple each equation by a different constant. As usual (see, e.g., Friedrichs, 1965) we introduce the linear condition

$$
\left(\mu^{(+)}, \mu_{0}^{(+)}\right)=\int_{-\infty}^{+\infty} \mu^{(+)}(x) \mu_{0}^{(+)}(x) d x=1
$$

which implies

$$
\left(\mu_{1}^{(+)}, \mu_{0}^{(+)}\right)=\left(\mu_{2}^{(+)}, \mu_{0}^{(+)}\right)=\ldots=0
$$

Now, the system $(30)-(32), \ldots$ can be successively solved with respect to $\mu_{1}^{(+)}, \mu_{2}^{(+)} ; \ldots$ The equation (31) is solvable if its right hand side is orthogonal to $\mu_{0}^{(+)}$. This gives the value

$$
\nu_{1}^{(+)}=\frac{\left[\nu_{0}^{(+)}\right]^{2}\left(\mu_{0}^{(+)}, C \mu_{0}^{(+)}\right)-1}{\left(\mu_{0}^{(+)}, B \mu_{0}^{(+)}\right)}
$$

where $\left(\mu_{0}^{(+)}, B \mu_{0}^{(+)}\right)>0$, since $B$ is a positive operator (see Appendix). Here (30) and the difinition of $A_{0}$ should be taken into account. When $\mu_{1}^{(+)}$is obtained, the in the same way $\nu_{2}^{(+)}$and $\mu_{2}^{(+)}$can be determined, and so on.

Thus, the eigenvalue $\nu^{(+)}$of the perturbed problem close to the positive eigenvalue $\nu_{0}^{(+)}$of the uperturbed problem can be found with any necessary accuracy. It is clear, that $\nu^{(+)}>0$, if $\epsilon$ is sufficiently small, and there is a finite set $\left\{\nu^{(+)}\right\}$of such positive eigenvalues.

Now, let us construct the expansions (29), starting from $\nu_{0}^{(0)}=0$, which is an eigenvalue of (30). Then, the system (31)-(32),.. can be rewritten as follows

$$
\begin{gathered}
\nu_{1}^{(0)} B \mu_{0}^{(0)}-\mu_{0}^{(0)}=0 \\
\nu_{1}^{(0)} B \mu_{1}^{(0)}-\mu_{1}^{(0)}=\left[\nu_{1}^{(0)}\right]^{2} A_{0} \mu_{0}^{(0)}-\mu_{0}^{(0)}-\nu_{2}^{(0)} B \mu_{0}^{(0)}
\end{gathered}
$$

In (34) the equation (33) is taken into account.

According to (33), we have usual eigenvalue problem to find a pair $\left(\nu_{1}^{(0)}, \mu_{0}^{(0)}\right)$. It has a finite set of positive eigenvalues if $k$ is large enough (see Appendix). Let us choose one non-degenerate eigenvalue $\nu_{1}^{(0)}$, and let $\mu_{0}^{(0)}$ be corresponding normalized eigenfunction. As above, we require

$$
\left(\mu^{(0)}, \mu_{0}^{(0)}\right)=1
$$


that yields

$$
\left(\mu_{1}^{(0)}, \mu_{0}^{(0)}\right)=\left(\mu_{2}^{(0)}, \mu_{0}^{(0)}\right)=\ldots=0
$$

This allows to solve successively the system $(34), \ldots$ with respect to $\mu_{1}^{(0)}, \mu_{2}^{(0)}, \ldots$

The equation (34) is solvable, if its right hand side is orthogonal to $\mu_{0}^{(0)}$. This gives the value

$$
\nu_{2}^{(0)}=\frac{\left[\nu_{1}^{(0)}\right]^{2}\left(\mu_{0}^{(0)}, A_{0} \mu_{0}^{(0)}\right)-1}{\left(\mu_{0}^{(0)}, B \mu_{0}^{(0)}\right)}
$$

where $\left(\mu_{0}^{(0)}, B \mu_{0}^{(0)}\right)>0$, because $B$ is a positive operator (see Appendix).

Since $\nu_{1}^{(0)}>0$, then

$$
\nu^{(0)}=\epsilon \nu_{1}^{(0)}+\epsilon^{2} \nu_{2}^{(0)}+\ldots>0
$$

if $\epsilon$ is small enough.

Thus, the following conclusions can be made

1) Under the assumptions that $k$ is large enough and $\epsilon$ is small the perturbed square pencil (22) has two finite sets of positive eigenvalues $\left\{\nu^{(+)}\right\}$and $\left\{\nu^{(0)}\right\}$.

2) If eigenvalues $\left\{\nu_{0}^{(+)}\right\}$of (24) are non-degenerate $\left(\left\{\nu_{0}^{(+)}\right\}\right.$is a finite set), then each of them induces a positive eigenvalue $\nu^{(+)}$of the form (29).

3) If characteristic values $\left\{\nu_{1}^{(0)}\right\}$ of (33) are non-degenerate $\left(\left\{\nu_{1}^{(0)}\right\}\right.$ is a finite set if $k$ is large enough), then each of them induces a positive eigenvalue $\nu^{(0)}$ of the form (35) for sufficiently small $\epsilon$.

\section{Discussion}

What is the hydrodynamical meaning of the eigenvalues obtained in the previous Section?

Since the eigenvalue $\nu^{(+)}$is close to the eigenvalue $\nu_{0}^{(+)}$and the last corresponds to trapped mode on the surface of homogeneous fluid without interface, then $\nu^{(+)}$corresponds to trapped mode of waves on the free surface of two-layer fluid.

The eigenvalue $\nu^{(0)}$ is proportional to $\epsilon$ and there are no such eigenvalues for the homogeneous fluid. Consequently, this eigenvalue corresponds to trapped mode of internal waves on the interface between two layers.

It is interesting to estimate the frequency of trapped mode of internal waves.

According to Theorem from Appendix $\nu_{1}^{(0)}$ is close to the characteristic value of the operator $2 V$ if $k$ is large enough. It was shown in the paper by Ursell (1987) that characteristic values of $V$ correspond to trapped modes of waves on the free surface of the lower fluid in absence of the upper fluid. If we denote by $\omega_{i}$ the frequency of trapped mode of internal 
waves and by $\omega_{s}$ the frequency of trapped mode of surface waves for lower fluid in absence of upper fluid, then we have approximate equality

$$
2 \omega_{i}^{2} \approx \omega_{s}^{2} \epsilon \quad \text { or } \quad \omega_{i} / \omega_{s} \approx(\epsilon / 2)^{1 / 2}
$$

which is valid if $k$ is large enough.

For three values of the parameter $\epsilon$, that describes some real interfaces between fresh and salt water one can find corresponding approximate quotients $\omega_{i} / \omega_{s}$ in the following table.

$$
\begin{array}{cccc}
\epsilon & 0.04 & 0.02 & 0.01 \\
\omega_{i} / \omega_{s} & 0.14 & 0.10 & 0.07
\end{array}
$$

This diminution of the frequency of trapped internal waves is similar to the decreasing of the velocity of internal waves comparing with the velocity of surface waves described in Art. 231 of Lamb's book (1932). In the last case the velocity ratio is exactly equal to $\epsilon /(2+\epsilon)$.

The method developed above in the case, when the lower fluid has infinite depth, is valid without any changes for the two-layer fluid with both layers of finite depth. One has only to take another Green's function on the second stage of reduction. Such a Green function for the fluid of finite depth was constructed by Ursell (1987).

The case when the cylinder is immersed totally in the upper layer is more complicated. This problem can be easily reduced to the boundary value problem, which involves only the potential $u^{*}$ for the upper layer. However, apart from the usual condition on the free surface

$$
u_{y}^{*}-\nu u^{*}=0 \text { when } y=1
$$

the spectral parameter $\nu$ will be included into a pseudo-differential operator in the boundary condition on the interface. Then we have to use another Green's function satisfying (36). Such kind of the Green function was also constructed by Ursell (1987), but this Green function depends on $\nu$. Hence, one obtains a spectral problem with an operator-function depending on $\nu$ in a way which is not so simple as square operator pencil.

\section{Acknowledgement}

The author thanks Dr. J. Grue for the posibility to visit the University of Oslo where this work had been completed.

\section{References}

Aranha, J. A. P. 1988 Existence and some properties of waves trapped by submerged cylinder. J. Fluid Mech. 192, 421-433.

Bonnet, A.-S. \& Joly, P. 1990 Mathematical and numerical study of trapping waves. The Fifth Int. Workshop on Water Waves and Floating Bodies. Manchester (ed. P. A. Martin), pp. 25-28. 
Callan, M. A. 1990 Trapping modes above non-cylindrical bodies. The Fifth Int. Workshop on Water Waves and Floating Bodies. Manchester (ed. P. A. Martin), pp. 29-33.

Callan, M. A., Linton, C. M. \& Evans, D. V. 1991 Trapped waves in two-dimensional waveguides. J. Fluid Mech. 229, 51-64.

Friedrichs, K. O. 1965 Perturbation of Spectra in Hilbert Space. Amer. Math. Soc., Providence, RI.

Friis, A., Grue, J. \& Palm, E. 1991 Application of Fourier transform to the second order 2D wave diffraction problem. M. P. Tulin's Festschrift: Mathematical Approaches in Hydrodynamics (ed. T. Muloh), pp. 209-227. SIAM.

Gradshtein, I. S. \& Ryzhik, I. M. 1965 Tables of Integrals, Series, and Products. Academic Press, New York.

Jones, D. S. 1953 The eigenvalues of $\nabla^{2} u+\lambda u=0$ when the boundary conditions are given on semi-infinite domains. Proc. Camb. Phil. Soc. 49, 668-684.

Kato, T. 1966 Perturbation Theory for Linear Operators. Springer, Berlin et al.

Kuznetsov, N. G. 1991 Uniqueness of a solution of a linear problem for stationary oscillation of a liquid. Diff. Equations 27, 187-194.

Lamb, H. 1932 Hydrodynamics. Cambridge University Press.

Martin, P. A. 1989 On the computation and excitation of trapping modes. The Fourth Int. Workshop on Water Waves and Floating Bodies. Univ. of Oslo, Dept. of Math. (eds. J. Grue \& E. Palm), 145-148.

McIver, P. 1991 Trapping of surface water waves by fixed bodies in a channel. Quart. J. Mech. Appl. Math. 33, 193-208.

McIver, P. \& Evans, D. V. 1985 The trapping of surface waves above a submerged horizontal cylinder. J. Fluid Mech. 151, 243-255.

Stokes, G. C. 1846 Report on recent researches in hydrodynamics. Brit. Assn Rep.

Ursell, F. 1951 Trapping modes in the theory of surface waves. Proc. Camb. Phil. Soc. 47, 347-358.

Ursell, F. 1987 Mathematical aspects of trapping modes in the theory of surface waves. $J$. Fluid Mech. 183, 421-437.

Vainberg, B. R. \& Maz'ya, V. G. 1973 On the problem of the steady state oscillations of a fluid layer of variable depth. Trans. Moscow Math. Soc. 28, 56-73.

Valdimirov, V. S. 1971 Equations of Mathematical Physics. Marcel Dekker, New York. 


\section{Appendix. Properties of the operators from Section 3.}

We first consider the properties of $C$ and $L$, since these operators have the explicit kernels. Proposition 1. The operators $C$ and $L$ both are symmetric, positive and bounded operators.

Proof. Due to Plancherel's theorem and to the definition of $C$, we have

$$
(C \mu, \mu)=2 \pi(\widetilde{C \mu}, \tilde{\mu})=\int_{-\infty}^{+\infty} \frac{\tilde{\mu}^{2}(\xi)}{k^{2}+\xi^{2}} d \xi
$$

Now, it is clear that $(C \mu, \mu)>0$, if $\mu \not \equiv 0$. Then $C$ is a positive, symmetric operator. Furthermore, $\left(k^{2}+\xi^{2}\right)^{-1} \leq k^{-2}$. Hence,

$$
(C \mu, \mu) \leq k^{-2}\|\mu\|^{2}
$$

what means that $C$ is a bounded operator.

In the same way

$$
(L \mu, \mu)=2 \pi(\widetilde{L \mu}, \tilde{\mu})=\int_{-\infty}^{+\infty} \frac{\operatorname{ctnh} \lambda}{\lambda} \tilde{\mu}^{2}(\xi) d \xi
$$

where $\lambda=\left(k^{2}+\xi^{2}\right)^{1 / 2}$. Then $L$ is a positive, symmetric operator.

Since

$$
\left(\frac{\operatorname{ctnh} \lambda}{\lambda}\right)^{\prime}=\frac{1}{\lambda \sinh ^{2} \lambda}-\frac{\operatorname{ctnh} \lambda}{\lambda^{2}}=-\frac{1}{\lambda^{2} \sinh ^{2} \lambda}\left(\frac{\sinh 2 \lambda}{2}-\lambda\right)<0
$$

then

$$
\frac{\operatorname{ctnh} \lambda}{\lambda} \leq \frac{\operatorname{ctnh} k}{k}
$$

and $L$ is a bounded operator, which norm does not exceed $k^{-1} \operatorname{ctnh} k$. The proof is complete. The operator $V$ was introduced and investigated by Ursell (1987). The properties of $V$ can be summarized in the following two propositions.

Proposition 2. The operator $V$ is a simmetric, positive and bounded operator in $L_{2}(-\infty,+\infty)$.

We denote by $\|V\|$ the norm of $V$ in $L_{2}(-\infty,+\infty)$. If we consider the spectral problem

$$
(V-\lambda I) \mu=0
$$

then we have

Proposition 3. The spectrum of $V$ is real, continuous for $0<\lambda<k^{-1}$ and discrete for $k^{-1}<\lambda<\|V\|$. Moreover, there exists at least one point eigenvalue.

From Propositions 1 and 2 immediately follows 
Corollary 1. Operators $A, A_{0}$ and $B$ are bounded and $B$ is a symmetric, positive.

This Corollary follows from the definition of $A, A_{0}$ and $B$.

Theorem. If $k$ is large enough, then there exists at least one positive point eigenvalue of the operator $B$. The number of positive point eigenvalues of $B$ is finite. These eigenvalues are close to the point eigenvalues of the operator $2 \mathrm{~V}$.

Proof. Let us write

$$
B=L+V=2 V+(L-V)
$$

Since the operators involved are symmetric, positive and bounded, then according to the general perturbation theory (see Kato, 1966) it is enough to show that the norm of the operator $L-V$ is small, if $k$ is large enough. Really, from the Proposition 3 it follows that the operator $2 V$ has a finite number (at least one) of positive point eigenvalues. Hence, if $L-V$ has a small norm, then the eigenvalues of $B$ exist and are positive and close to the eigenvalues of $2 \mathrm{~V}$.

The kernel of $L$ can be represented in the form

$$
\mathrm{E}(k|x|)=\frac{1}{\pi} \int_{0}^{\infty} \frac{\operatorname{ctnh} \lambda}{\lambda} \cos x \xi d \xi=\frac{1}{\pi} \int_{0}^{\infty} \frac{\cos x \lambda}{\lambda} d \xi+\frac{2}{\pi} \int_{0}^{\infty} \frac{\cos x \xi}{\lambda} \frac{e^{-2 \lambda}}{1-e^{-2 \lambda}} d \xi
$$

Due to the formula 6.671.14 from Gradshtein \& Ryzhik (1965) we have for the Fourier transform of the McDonald function

$$
2 \int_{0}^{\infty} K_{0}(k x) \cos x \xi d x=\pi /\left(k^{2}+\xi^{2}\right)^{1 / 2}=\pi / \lambda
$$

Comparing this formula with the previous one, we find

$$
\mathrm{E}(k|x|)=\pi^{-1} K_{0}(k|x|)+N(k, k|x|)
$$

where

$$
N(k, k|x|)=\frac{2}{\pi} \int_{0}^{\infty} \frac{\cos x \xi}{\lambda} \frac{e^{-2 \lambda}}{1-e^{-2 \lambda}} d \xi
$$

Thus, the operator $L-V$ has the kernel

$$
N(k, k|x-\sigma|)-\pi^{-1} M(x, \sigma)
$$

Here we also used (20). We shall demonstrate that each of the operators with kernels $N$ and $M$ is small if $k$ is large enough.

Since the kernel $N$ depends on $|x-\sigma|$, it is convenient to apply Plancherel's theorem. Then, we have

$$
\int_{-\infty}^{+\infty} \mu(x) d x \int_{-\infty}^{+\infty} N(k, k|x-\sigma|) \mu(\sigma) d \sigma=\int_{-\infty}^{+\infty} \frac{2}{\lambda} \frac{e^{-2 \lambda}}{1-e^{-2 \lambda}} \tilde{\mu}^{2}(\xi) d \xi
$$


Considering the function

$$
f(x)=\frac{e^{-x}}{x\left(1-e^{-x}\right)}
$$

we conclude that it is monotonically decreasing for $x>0$, since

$$
f^{\prime}(x)=\frac{-x e^{-x}\left(1-e^{-x}\right)-e^{-x}\left[\left(1-e^{-x}\right)+x e^{-x}\right]}{x^{2}\left(1-e^{-x}\right)^{2}}<0
$$

Hence, the norm of the operator with the kernel $N(k, k|x-\sigma|)$ does not exceed the value

$$
\frac{2}{k} \frac{e^{-2 k}}{1-e^{-2 k}}
$$

which is small, if $k$ is large enough.

Now, let us estimate the norm of the operator with the kernel $M(x, \sigma)$. Due to the Schwarz inequality we can write

$$
\int_{-\infty}^{+\infty} d x\left(\int_{-\infty}^{+\infty} M(x, \sigma) \mu(\sigma) d \sigma\right)^{2} \leq\|\mu\|^{2} \int_{-\infty}^{+\infty} \int_{-\infty}^{+\infty} M^{2}(x, \sigma) d x d \sigma
$$

Then according to (20) and the Schwarz inequality

$$
\begin{aligned}
& \int_{-\infty}^{+\infty} \int_{-\infty}^{+\infty} M^{2}(x, \sigma) d x d \sigma \\
& \leq 4\left\{\int_{-\infty}^{+\infty} d \sigma \int_{S} m^{2}(s, \sigma) d s\right\}\left\{\int_{-\infty}^{+\infty} d x \int_{S}\left[K_{0}\left(k\left\{(x-X(s))^{2}+Y^{2}(s)\right\}^{1 / 2}\right) d s\right]\right\}
\end{aligned}
$$

Hence the norm of the operator with the kernel $M(x, \sigma)$ is small for large enough values of $k$, because of asymptotic behaviour of the McDonald function at infinity. Here we used the fact that the distance between $S$ and the interface is positive. The proof is complete. 
